\title{
P02-4-27 Poster session
}

\section{Possible involvement of TRPM4 channels in the cholinergic contractile responses in mouse detrusor and ileal smooth muscles}

\author{
Firoj Alom ${ }^{1}$, Hayato Matsuyama ${ }^{2}$, Hiroshi Nagano ${ }^{1}$, Yasuyuki Tanahashi ${ }^{3}$, Toshihiro Unno ${ }^{2}$ \\ ${ }^{I}$ Department of Pathogenetic Veterinary Science, United Graduate School of Veterinary Science, Gifu University, \\ Japan, ${ }^{2}$ Laboratory of Veterinary Pharmacology, Department of Veterinary Medicine, Faculty of Applied Biological \\ Science, Gifu University, Japan, ${ }^{3}$ Department of Animal Medical Sciences, Faculty of Life Sciences, Kyoto Sangyo \\ University, Japan
}

Background: In visceral smooth muscle cells, opening of several types of cationic channels are involved in contractile responses induced by receptor activation. Recently, transient receptor potential melastatin 4 (TRPM4) channels have been suggested to mediate contractions of a certain type of smooth muscle. In the present study, we investigated effects of 9-phenanthrol, a novel, specific inhibitor of TRPM4 channels, on contractile responses induced by an electrical field stimulation (EFS) or a muscarinic agonist to explore the involvement of TRPM4 channels in cholinergic contractile responses in mouse detrusor and ileal smooth muscles.

Methods: Urinary bladder and ileal part of intestine were isolated from mice of either sex (2-11 months old), and detrusor and ileal longitudinal muscle strips were prepared for isometric tension recordings. Cholinergic contractions were recorded by application of carbachol or EFS (pulse width: $0.5 \mathrm{~ms}$, strength: $50 \mathrm{~V}$, frequency: 1-100 Hz) for $5 \mathrm{~s}$. Alpha, beta-methylene ATP (50 micromole) was applied to bath solution (Tyrode solution) to inhibit purinergic component for the recording of detrusor contraction, and guanethidine (1 micromole) and L-NAME (100 micromole) were treated to inhibit adrenergic and nitrergic components for the recording of ileal contraction. In some experiments, intracellularly released calcium-mediated contractions induced by carbachol (10 micromole) were recorded in the presence of calciumfree, $140 \mathrm{mM} \mathrm{KCl}$ Tyrode solution.

Results: EFS elicited a transient detrusor contraction in frequency-dependent manner, and application of atropine or tetrodotoxin abolished the transient contraction. 9-phenanthrol (3-30 micromole) significantly inhibited EFS-induced or carbachol-induced cholinergic detrusor contractions. However, 9-phenanthrol (10 micromole) had no significant inhibitory effect on the intracellularly released calcium-mediated and $70 \mathrm{mM} \mathrm{KCl-induced} \mathrm{detrusor} \mathrm{contractions.} \mathrm{In} \mathrm{ileal}$ longitudinal muscle preparations, EFS elicited a transient, atropine-sensitive contraction in frequency-dependent manner, and 9-phenanthrol (30 micromole) also significantly inhibited EFS-induced or carbachol-induced cholinergic ileal contractions without affecting $70 \mathrm{mM} \mathrm{KCl}$-induced contraction.

Conclusion: These results suggest that muscarinic receptor activation stimulates the opening of TRPM4 channels to produce membrane depolarization and then induces muscle contraction in mouse detrusor and ileal longitudinal smooth muscles. 